\title{
Hyperbilirubinemia during atazanavir treatment in people living with HIV (PLHIV), Sri Lanka.
}

\author{
D.I.Rajapaksha ${ }^{1}$, A.Azraan ${ }^{2}$, L.I.Rajapaksa ${ }^{3}$
}

\begin{abstract}
Introduction: Atazanavir (ATV) is an antiretroviral drug of the protease inhibitor class. It is used to treat HIV infection in combination with other HIV medications. Atazanavir was first introduced to National STD/AIDs control programme of Sri Lanka in 2014. Symptomatic hyperbilirubinemia is a common adverse effect associated with atazanavir use. Objective of this study was to see the significance of hyperbilirubinemia with ritonavir boosted atazanavir use among people living with HIV receiving antiretroviral therapy.
\end{abstract}

Method: This was a descriptive cross sectional study carried out among all PLHIV started on retonavir boosted atazanavir based regimens, who were receiving care services at National STD/AIDS control programme, Sri Lanka.

Results: Total of 40 PLHIV who had taken atazanavir more than 90 days during the study period (28 months) were analyzed. Cumulative incidence of hyperbilirunemia of grade 2 or above during the study period was $40 \%(n=16)$. Almost all PLHIV had isolated indirect hyperbilirubinemia.

Conclusions: Significant proportion of patients (40\%) developed grade 2 or more indirect hyperbilirubinemia following initiation of atazanavir based therapy and $25 \%(n=10)$ improved symptomatically and biochemically during follow up. But in $12.5 \%(n=5)$ patients antiretroviral regimen had to be substituted.

Key words: Atazanavir, hyperbilirubinemia, substitution, People living with HIV (PLHIV)

Authors: corresponding author; ${ }^{1}$ Dr D.I. Rajapaksha, MBBS, PgDVen, MD Venereology; Senior Registrar, NSACP, Colombo, Sri Lanka. Email: irukamfc@yahoo.com

${ }^{2} \operatorname{Dr}$ A. Azraan, MBBS, PgDVen, MD Venereology; Senior Registrar, NSACP, Colombo, Sri Lanka.

${ }^{3}$ Dr L. I. Rajapaksa, MBBS, Dip STD, MSc Community Medicine, MD Community Medicine; Consultant Venereologist, NSACP, Colombo, Sri Lanka.

Acknowledgement: HIV clinic staff

Conflict of interest: No conflict of interest.

Funding: No funding support for this study

Originality: This is an original work, not published or presented anywhere.

Submitted: 29.11.2016, Accepted: 15.12.2016

\section{Full article}

neucleoside reverse transcriptase inhibitor or an integrase inhibitor as first line

\section{Introduction}

World Health Organization (WHO) consolidated guidelines on the use of antiretroviral drugs for treating and preventing HIV infection 2016, recommends two nucleoside/ nucleotide reverse transcriptase inhibitors plus a non antiretroviral therapy (ART). National guideline of antiretroviral drugs for treating and prevention of HIV infection 2014, Sri Lanka prefer tenofovir (TDF), emtricitabine (FTC) plus efavirenz (EFV) as first line ART regimen. There are instances, where we use protease inhibitor class in first line instead of efavirenz in some patients such as patients 
with psychiatric illness, history of suicidal attempts and who are engaged in shift duties especially night tasks, etc. When first line antiretroviral regimen failed, we prefer second line regimen constitute with a boosted protease inhibitor. In Sri Lanka, protease inhibitor class is also used as a switching drug to people who cannot tolerate non nucleoside reverse transcriptase inhibitor class. Ritonavir boosted atazanavir (ATV/r), lopinavir (LPV/r) and darunavir (DRV/r) are the available options in Sri Lanka. Darunavir is mostly restricted to third line regimens. Following introduction of atazanavir (ATV) in 2014 in Sri Lanka, it is more preferred due to once daily dosing and less likely to cause lipid elevations. Atazanavir is the first protease inhibitor approved for once daily dosing. It is available in 300mg capsule formulation. It was first approved in 2003 from U.S. Food and Drug Administration. It is recommended for treatment naive and treatment experienced HIV patients (1) (2).

Common side effects of atazanavir include nausea, vomiting, jaundice, abdominal pain, diarrhea, dizziness and flu like illness. Atazanavir associated hyperbilirubinemia is a common adverse effect and it was defined as hyperbilirubinemia developing after initiation of atazanavir therapy in the absence of other causes of hyperbilirubinemia (1). According to WHO consolidated guidelines on the use of antiretroviral drugs for treating and preventing HIV infection 2016, indirect hyperbilirubinemia (clinical jaundice) is a major type of toxicity associated with ritonavir boosted atazanavir (ATV/r) therapy. But this phenomenon is clinically benign but potentially stigmatizing. WHO consolidated guidelines 2016, mentioned to substitute only if adherence is compromised.

Hyperbilirubinemia as an ART toxicity was classified in accordance with WHO consolidated guidelines 2016 (2) (Table 1).
Table 1: Severity grading of hyperbilirubinemia according to the laboratory parameters

\begin{tabular}{ll}
\hline Toxicity & Hyperbilirubinemia \\
\hline Grade 1 & $>1.0-1.5 \times U L N$ \\
\hline Grade 2 & $>1.6-2.5 \times U L N$ \\
\hline Grade 3 & $>2.6-5 \times U L N$ \\
\hline Grade 4 & $>5 \times U L N$ \\
\hline
\end{tabular}

Atazanavir causes an elevation of unconjugated hyperbilirubinemia as a result of UDP glucuronyl transferase $1 \mathrm{~A} 1$ inhibition. UGT $1 A 1$ is responsible liver enzyme for conjugation of bilirubin to soluble glucuronide (1). This adverse effect appears with initiation of atazanavir. Most frequent symptoms are scleral icterus and yellowish discolouration of skin. This is mostly isolated hyperbilirubinemia and other liver enzymes are not affected.

Following introduction of atazanavir to national programme since 2014 we have started atazanavir in combination with other antiretrovirals for 42 people living with HIV. However, little is known about atazanavir induced hyperbilirubinemia among Sri Lankan patients. The objective of the study was to assess significance of hyperbilirubinemia in HIV infected patients started on atazanavir based regimen.

\section{Method}

A descriptive cross sectional study was conducted among all the PLHIV who initiated antiretroviral therapy (ART) with atazanavir (300mg per day) from May 2014 to August 2016 (28 months) and received care services at the Colombo, HIV clinic of the National STD/ AIDS control programme, Sri Lanka. PLHIV who were started on atazanavir based regimen at least 90 days prior to study period were included in the study. Forty (40) study subjects were included in the study. Atazanavir exposure was evaluated from records of all patients. The prescription of antiretroviral with the starting and ending dates were identified through patient's 
records. Baseline liver profile tests, hepatitis $B$ surface antigen and hepatitis $C$ antibody screening test, which were carried out prior to initiation of antiretroviral therapy were studied and documented. Liver profiles were followed up at regular intervals of 1 month, 3 months, 6 months and thereafter in every 6 months following initiation of atazanavir. The total bilirubin level recorded at any point during the follow up was taken in to account in deciding the severity grading of toxicity. Data was collected using a data extraction sheet and data was processed and analyzed by using Microsoft excel 2013.

\section{Results}

In the study population ( $n=40)$, majority were males (68\%). An overall mean CD4 cell count at the initiation of atazanavir was 481 cells $/ \mu \mathrm{l}$ (Mdn=378 cells/ $\mu \mathrm{l})$ and the range was $16-$ 960 cells $/ \mu$ l.

Distribution of other baseline characteristics is tabulated in table 1.

Table 1: Baseline characteristics

\begin{tabular}{|c|c|c|c|}
\hline Category & Category & No. & Percent \\
\hline \multirow[t]{4}{*}{ Regimen } & $T D F+F T C+A T V / r$ & 38 & $95 \%$ \\
\hline & $A B C+3 T C+A T V / r$ & 1 & $2.5 \%$ \\
\hline & $Z D V+3 T C+A T V / r$ & 1 & $2.5 \%$ \\
\hline & Total & 40 & $100 \%$ \\
\hline \multirow{6}{*}{$\begin{array}{l}\text { ATV/r } \\
\text { started } \\
\text { among }\end{array}$} & Naïve & 21 & $52.5 \%$ \\
\hline & $\begin{array}{ll}\text { First } & \text { line } \\
\text { substitution }\end{array}$ & 7 & $17.5 \%$ \\
\hline & second line & 10 & $25 \%$ \\
\hline & $\begin{array}{l}\text { Second line } \\
\text { substitution }\end{array}$ & 1 & $2.5 \%$ \\
\hline & Third line & 1 & $2.5 \%$ \\
\hline & Total & 40 & $100 \%$ \\
\hline \multirow[t]{3}{*}{$A L T$} & $<U L N$ & 36 & $90 \%$ \\
\hline & $B / w 1-2 \times U L N$ & 4 & $10 \%$ \\
\hline & Total & 40 & $100 \%$ \\
\hline \multirow[t]{3}{*}{$A S T$} & $<U L N$ & 36 & $90 \%$ \\
\hline & $B / w 1-2 \times U L N$ & 4 & $10 \%$ \\
\hline & Total & 40 & $100 \%$ \\
\hline \multirow[t]{3}{*}{$G G T$} & $<U L N$ & 33 & $82.5 \%$ \\
\hline & $B / w 1-2 \times U L N$ & 7 & $17.5 \%$ \\
\hline & Total & 40 & $100 \%$ \\
\hline S.bilirubin & $<U L N$ & 40 & $100 \%$ \\
\hline$H B s A g$ & Negative & 40 & $100 \%$ \\
\hline
\end{tabular}

\begin{tabular}{llcr}
\hline$H C A b$ & Negative & 40 & $100 \%$ \\
\hline \multirow{2}{*}{$\begin{array}{l}\text { Alcohol } \\
\text { abuse }\end{array}$} & Yes & 13 & $32.5 \%$ \\
\cline { 2 - 4 } & No & 27 & $67.5 \%$ \\
\cline { 2 - 4 } & Total & 40 & $100 \%$ \\
\hline \multirow{2}{*}{$\begin{array}{l}\text { INAH } \\
\text { prophylaxis }\end{array}$} & Yes & 14 & $35 \%$ \\
\cline { 2 - 4 } & No & 26 & $65 \%$ \\
\cline { 2 - 4 } & Total & 40 & $100 \%$ \\
\hline $\begin{array}{l}\text { B/w-Between, } \\
\text { Isoniacid }\end{array}$ & ULN-Upper limit of & normal, & INAH- \\
& & &
\end{tabular}

As baseline, alanine transaminase (ALT), aspartate transaminase (AST), alkaline phosphatase (ALP), gamma glutamyl transferase (GGT) and serum bilirubin levels were measured prior to the initiation of atazanavir/ritonavir based therapy. All the study participants had total bilirubin levels less than upper limit of normal and liver enzyme levels less than twice the upper limit normal (ULN) before starting atazanavir. All the study subjects had been tested for hepatitis $B$ and $C$ coinfections prior to initiation of therapy and none were positive. (Table 1)

They were followed up at monthly intervals and liver profiles repeated at 1 month, 3 months and 6 months after the initiation of antiretroviral therapy. Thereafter, in every 6 months if there were no other indications.

Cumulative incidence of hyperbilirunaemia (toxicity grade 2 or above) during the period of 28 months was $40 \%(n=16)$ and of them $35 \%(n=14)$ were symptomatic with scleral icterus. In all cases hyperbilirubinemia was developed within first 90 days of initiation of therapy.

Among those who developed indirect hyperbilirubinemia, none showed elevations of AST, ALT, GGT, ALP more than twice the upper limit of normal. Hyperbilirubinemia due to unconjugated hyperbilirubinemia was seen among all study subjects.

During the study period, outcome of interest (total bilirubin level) was measured and recorded against the level of toxicity grades (table 2) 
Table 2: Distribution by level of toxicity

\begin{tabular}{llrr}
\hline Toxicity & Bilirubin & No & Percent \\
\hline No toxicity & $<$ ULN & 20 & $50 \%$ \\
\hline Grade 1 & $>1.0-1.5 \times$ ULN & 4 & $10 \%$ \\
\hline Grade 2 & $>1.6-2.5 \times$ ULN & 5 & $12.5 \%$ \\
\hline Grade 3 & $>2.6-5 \times$ ULN & 6 & $15 \%$ \\
\hline Grade 4 & $>5 \times$ ULN & 5 & $12.5 \%$ \\
\hline Total & & 40 & $100 \%$ \\
\hline
\end{tabular}

Patients with grade 2 toxicity ( 5 patients) continued with the same regimen, hyperbilirubinemia improved with time. Six patients developed symptomatic grade 3 toxicity with scleral icterus. Among them one patient had ultrasonic evidence of grade 2 fatty liver disease since baseline and she was on long term antidepressants in addition to antiretrovirals. Patient was really worried about icterus and atazanavir discontinued due to cosmetic reasons. Her regimen was substituted with boosted lopinavir and she improved rapidly. Four patients with grade 3 toxicity continued on same regimen with close observation of symptoms and liver functions. They showed gradual reduction of total bilirubin level and regression of symptoms with time. One patient with symptomatic adverse effects lost to follow up. Grade 4 toxicity developed in 5 patients. They had isolated indirect hyperbilirubinemia. Atazanavir was discontinued in 4 patients with grade 4 toxicity.

Cosmetic issues were a concern for PLHIV as they had deep scleral icterus. And it was substituted with another protease inhibitor or non nucleoside reverse transcriptase inhibitor due to aesthetic reasons. Following substitution, they improved rapidly where symptoms disappeared and liver biochemistry returned to normal levels.

Three (7.5\%) patients who were started on atazanavir experienced virological failure due to adherence issues and had to be started on another antiretroviral regimen. It was substituted in one PLHIV to avoid drug interactions with inhalational steroids, as she was on stage 3 management level of bronchial asthma.

\section{Discussion}

Several clinical studies demonstrated atazanavir induced hyperbilirubinemia. CASTLE was a randomized 96 weeks trial which demonstrated clinical significance of hyperbilirubinemia among HIV 1 infected patients treated with ritonavir boosted atazanavir. Overall $44 \%$ of patients receiving atazanavir/ritonavir had hyperbilirubinemia (grade 3-4) at any time during the 96 weeks study. Less than $1 \%$ of patients discontinued atazanavir due to hyperbilirubinemia, jaundice or scleral icterus (3). Atazanavir and other determinants of hyperbilirubinemia studied in a cohort of 1150 HIV positive patients of 9 year follow up period in Canada. Cumulative incidence of hyperbilirubinemia of grade 3 and 4 after 1,5 and 8 years exposure to atazanavir were $30.1 \%, 73.4 \%$ and $83.6 \%$, respectively (4). Findings among PLHIV in Sri Lanka were also supported by CASTLE and atazanavir and other determinants cohort study of 1150 patients in Canada.

This study provided information on use of atazanavir among Sri Lankan PLHIV. All the patients started on atazanavir based therapy since 2014 were included in the study. In this group 3 PLHIV had poor adherence and experienced virological failure while another PLHIV defaulted for follow up. Cumulative incidence of hyperbilirubinemia during 28 months period was $40 \%$. Atazanavir associated hyperbilirubinemia was not related with elevations of other liver enzymes, but it led to discontinuation in 5 patients due to cosmetic reasons. However, there were few limitations which need to be mentioned. Firstly, small sample size is a concern. Monitoring of liver functions could be done at more regular intervals, if it was available consistently. 


\section{Conclusion}

Significant proportion of study subjects (40\%) developed isolated indirect hyperbilirubinemia during the period studied (28 months). However, none showed elevations of liver enzymes more than twice the upper limit normal. Cosmetic issues were a concern among study subjects and led to discontinuation of therapy in $12.5 \%$. As our sample size is small we cannot conclude suitability of atazanavir as a first line therapy. We need to follow up larger cohort of patients to predict about adverse effects and outcome of atazanavir use.

\section{References}

1. Drug record - atazanavir. United States: National Library of Medicine

(https://livertox.nih.gov/Atazanavir/, accessed 25 November 2016).

2. Consolidated guidelines on the use of antiretroviral drugs for treating and preventing HIV infection, recommendations for a public health approach. Geneva: World Health Organization; second edition 2016

(http://www.who.int/hiv/pub/arv/arv-2016/en/, accessed 21 November 2016).

3. Molina JM, Andrade-Villanueva J, Echevarria et al; management of antiretroviral naïve HIV 1 infected patients: 96 week efficacy and safety results of the CASTLE

study;2010(https://www.ncbi.nlm.nih.gov/pubmed /20032785, accessed on 15 November 2016).

4. Claudie L, Jean-Guy B, Helen T, et al; atazanavir and other determinants of hyperbilirubinemia in a cohort of 1150 HIV - positive patients: Results from 9 years of follow up; 2013 (https://www.ncbi.nlm.nih.gov/pmc/articles/PMC3 704117, accessed on 18 November 2016). 